\title{
Molecularly Imprinted Polypyrrole on Glassy Carbon Electrode Modified with Reduced Graphene Oxide and Gold Nanoparticles for Isoamyl Alcohol Analysis in Fusel Oil
}

\author{
Thiago M. Mariano, ${ }^{\oplus a}$ Maísa A. Beluomini ${ }^{\oplus a}$ and Nelson R. Stradiotto ${ }^{\circledR} * a, b$ \\ anstituto de Química, Universidade Estadual Paulista (Unesp), 14800-060 Araraquara-SP, Brazil \\ ${ }^{b}$ Instituto de Pesquisa em Bioenergia, Universidade Estadual Paulista (Unesp), \\ 14800-060 Araraquara-SP, Brazil
}

\begin{abstract}
A molecularly imprinted electrochemical sensor for determination of isoamyl alcohol was successfully constructed by modifying the glassy carbon electrode (GCE) with gold nanoparticles (AuNPs) and reduced graphene oxide (RGO). The modification of the GCE increased the electron transfer rate and the electrode surface area, which, consequently, made more room available for the formation of the molecularly imprinted polymer (MIP). The development of the MIP on the modified surface was carried out via electropolymerization of the pyrrole in acetate buffer solution in the presence of the target molecule. The MIP-AuNPs-RGO/GCE showed low limit of detection $\left(8.4 \times 10^{-8} \mathrm{~mol} \mathrm{~L}^{-1}\right)$, satisfactory quantification range, amperometric sensitivity of $1.1 \mathrm{~A} \mathrm{~L} \mathrm{~mol}^{-1}$, excellent reproducibility and stability. Even when in the presence of analogous molecules, the sensor exhibited excellent selectivity. These results suggest that the proposed sensor is suitable for the detection of isoamyl alcohol in fusel oil samples.
\end{abstract}

Keywords: molecularly imprinted polypyrrole, reduced graphene oxide, gold nanoparticles, isoamyl alcohol, fusel oil

\section{Introduction}

The search for alternative fuels has become inevitable and urgent in the deeply seek to reduce the environmental impacts associated with the use of fossil fuels. In this sense, bioethanol plants and their byproducts have grown significantly in recent years as vital alternatives for lessening the use and even replacing fossil fuels and other harmful chemical substances employed as sources of energy. ${ }^{1}$ Bioethanol has proved to be economically useful in addition to being renewable. These are relevant features that explain the fact that it is being internationally marketed to satisfy countries with limited biomass resources.

The production of bioethanol generates byproducts such as fusel oil, obtained after fermentation and distillation of biomass, composed mainly of higher alcohols containing three or more carbon atoms, including isoamyl alcohol, isobutanol, propanol, butanol, among others. ${ }^{2,3}$ The fact that higher alcohols present in fusel oil are considered natural products provides these alcohols high commercial values.

*e-mail: nrstradi@gmail.com
Since these alcohols are high octane and low in exhaust gas emissions, they occupy an important place among alternative fuels. The separation of these compounds is regarded essentially important, since the market value of fusel oil is directly related to the amount of higher alcohols, especially of isoamyl alcohol. In a commercial plant, for example in Brazil, the yield of the fusel oil may vary from 1 to $11 \mathrm{~L}$ per $1000 \mathrm{~L}$ of alcohol produced, depending on the fermentation and distillation conditions as well as on the amount of nitrogen. ${ }^{4}$

Chromatographic methods are the predominant mechanism employed when it comes to the determination of fusel oil constituents in the literature. Pérez et al. ${ }^{5}$ quantified fusel oil constituents by gas chromatography for alcohols and esters, and liquid chromatography for carbonyl compounds using fusel oil samples from 3 different plants. The isoamyl (390 $\left.\mathrm{g} \mathrm{L}^{-1}\right)$, isobutyl $\left(158 \mathrm{~g} \mathrm{~L}^{-1}\right)$, ethyl (28.4 $\left.\mathrm{g} \mathrm{L}^{-1}\right)$, methyl $\left(16.6 \mathrm{~g} \mathrm{~L}^{-1}\right)$ and $n$-propyl $\left(11.9 \mathrm{~g} \mathrm{~L}^{-1}\right)$ alcohols represent approximately $77 \%$ of the fusel oil composition. Neale ${ }^{6}$ used high performance liquid chromatography to determine ethanol, isoamyl alcohol, isobutyl alcohol and L-propanol in alcoholic beverages. 
The disadvantages of these chromatographic methods lie in the high cost of maintenance, longer time of analysis and sample preparation. It, thus, makes sense to find a better method for the detection of these alcohols in fusel oil.

In this context, the use of electrochemical sensors for analysis is a suitable alternative method, as the production of these sensors comes at a low cost, apart from the fact that they do not require sample preparation, involve shorter analysis time compared to other analytical techniques, and exhibit high sensitivity and low limits of detection. To date, as stated by LaCourse et al., ${ }^{7}$ with the exception of hyphenated techniques which have been applied for the detection of several aliphatic alcohols via liquid chromatography using pulsed amperometric detection, no method has been published in the literature for the analysis of alcohol in fusel oil using direct electrochemical techniques. To the best of our knowledge, there is no specific sensor that has been reported in the literature for the determination of isoamyl alcohol in fusel oil samples.

Sensors based on molecularly imprinted polymer (MIPs) have been extensively used in electroanalysis because of their high selectivity and affinity for the imprinted molecule. This is mainly due to the high selectivity achieved by the predefined cavity formation of the target molecule in relation to similar compounds, ${ }^{8}$ which may also be able to differentiate enantiomeric molecules. ${ }^{9}$ This can be attributed to the preparation conditions of the sensor, where electropolymerization is carried out in the presence of the molecule of interest. This interaction between a functional monomer and the target molecule can be performed chemically or electrochemically through electropolymerization. The electropolymerization process has the advantage of being a simple, fast technique capable of forming a bonding film in addition to being able to control its thickness. Polypyrrole (PPy) has, for many years, been recognized as one of the most promising polymers for advanced sensors owing to its stability, biocompatibility, conductivity and easy preparation. In addition, PPy can be electropolymerized on several materials, where it can be found exhibiting electrochemical activity in neutral $\mathrm{pH}$ solution. Another very attractive property of PPy is its ability to incorporate anionic counterions during its synthesis by electropolymerization. PPy can easily be imprinted with template molecules. ${ }^{10}$

The sensitivity and selectivity of the MIP-based sensors are directly related to the quantity and quality of the wells actually imprinted on the surface of the sensor. ${ }^{11}$ Thus, bare electrodes are not of interest for the electropolymerization of MIP due to lack of accessibility to local imprinting, agglomeration of the wells during synthesis and low kinetics during the polymerization process. Furthermore, the adhesion of the polymer to the substrate is often low; this causes low electrochemical signal.

In this sense, the use of nanostructures has become a suitable way to overcome these shortcomings. In comparison to flat surfaces, nanomaterial-modified electrode surfaces have high volume/surface ratio, which increases the number of sites available for molecular imprinting, increasing both the binding capacity of MIP and its sensitivity, which can be up to 15 times greater than that of flat surfaces with similar imprint. ${ }^{12}$

In this context, graphene-based materials have attracted considerable attention due to their large surface area, high thermal and mechanical properties and high conductivity. Graphene oxide (GO) has been shown to be the most promising graphene derivative for the modification of vitreous carbon electrodes (GCE), mainly because it is soluble in water and contains functionalized oxygenated functional groups. With the electrochemical reduction of the GO directly on the surface of the electrode, it is possible to control the amount of the deposited material, forming reduced graphene oxide (RGO). The RGO on the surface of the sensor exhibits better electrical conductivity compared to the GO, due to the low quantity of oxygenated groups and the restoration in large numbers of the conjugated $\mathrm{sp}^{2}$ bonds. ${ }^{13}$ The high performance of MIP-based sensors on surfaces modified with graphene was evidenced by El Jaouhari et al., ${ }^{14}$ who used a GCE modified with RGO and zeolitic imidazolate framework to determine routine in orange juice samples, reaching low limits of detection.

Additionally, metallic nanoparticles (NPs) offer excellent electrocatalytic performance and high electrical conductivity. These properties provide better electrochemical response (higher current response) to the sensor, making these materials ideal for the preparation of chemical and biological sensors. ${ }^{15-17} \mathrm{~A}$ further point worth pointing out is that the introduction of NP on RGO sheets causes a greater increase in the surface area of the sensor, improving the analytical performance, such as sensitivity, due to the efficiency of electron transfer. ${ }^{18}$

In this study, a novel sensor was constructed based on molecular imprinting of polypyrrole on glassy carbon electrode modified with reduced graphene oxide and gold nanoparticles (AuNPs) for the sensitive determination of isoamyl alcohol. AuNPs-RGO was introduced in the method with the aim of improving the electrochemical signal and recognition capacity of the sensor for the analyte detection through an increase in number of imprinted sites per unit of specific surface area of the MIP sensor. Isoamyl alcohol and pyrrole were used as template and functional monomer, respectively. Subsequently, the isoamyl alcohol is removed from the polymer matrix, leading to the generation 
of cavities tailored for further recognition. All the steps involving the development of the electrode were carried out electrochemically and under the optimized conditions, the prepared sensor showed high recognition toward isoamyl alcohol. The applicability of the MIP-AuNPs-RGO/GCE in real samples was demonstrated by successfully quantifying isoamyl alcohol concentration in fusel oil.

\section{Experimental}

\section{Materials and instruments}

Pyrrole (purity: $\geq 98 \%$ ), isoamyl alcohol (purity: $\geq 98 \%$ ), potassium ferrocyanide $\left(\mathrm{K}_{4}\left[\mathrm{Fe}(\mathrm{CN})_{6}\right]\right.$ purity: $\left.99 \%\right)$, chloroauric acid (purity: 99\%) and graphene oxide (GO, purity: > 95\%) were purchased from Sigma-Aldrich (St. Louis, USA). Potassium chloride, potassium dihydrogen phosphate, dipotassium hydrogen phosphate, sodium acetate were employed as supporting electrolyte. Acetonitrile-acetic acid 5:1 (v/v) was used to extract the template from the MIP matrix. Solution of $1.0 \times 10^{-3} \mathrm{~mol} \mathrm{~L}^{-1}$ isoamyl alcohol and Py were prepared in $0.10 \mathrm{~mol} \mathrm{~L}^{-1}$ acetate buffer solution under $\mathrm{pH}=3.6$ for electropolymerization. The oxidation probe solution employed was $1.0 \times 10^{-3} \mathrm{~mol} \mathrm{~L}^{-1} \mathrm{~K}_{4}\left[\mathrm{Fe}(\mathrm{CN})_{6}\right]$ in $0.10 \mathrm{~mol} \mathrm{~L}^{-1} \mathrm{KCl}$. All the solutions were prepared with ultrapure water. Electrochemical voltammetric measurements were performed using a potentiostat Autolab PGSTAT30 coupled to a microcomputer that records and stores data obtained using the control software Nova 1.11. A conventional electrochemical cell with three electrodes was used. $\mathrm{Ag} / \mathrm{AgCl}\left(\mathrm{KCl} 3.0 \mathrm{~mol} \mathrm{~L}^{-1}\right)$ was used as reference electrode, platinum wire as the auxiliary electrode, and a GCE with diameter of $3.0 \mathrm{~mm}$ as working electrode. To undertake the measurements via differential pulse voltammetry (DPV), the following conditions were applied: pulse amplitude of $50 \mathrm{mV}$, pulse time of $100 \mathrm{~ms}$, potential step of $2.0 \mathrm{mV}$ and scan rate of $10 \mathrm{mV} \mathrm{s}^{-1}$. All experiments were carried out at room temperature.

\section{Cleaning procedure for GCE}

The GCE electrode was polished with $0.30 \mu \mathrm{m}$ alumina powder using a felt and electrochemically polished by successive scans at potential range of -0.50 to $+1.5 \mathrm{~V}$ in $0.50 \mathrm{~mol} \mathrm{~L}^{-1} \mathrm{H}_{2} \mathrm{SO}_{4}$ at $50 \mathrm{mV} \mathrm{s}^{-1}$ until voltammogram cyclic characteristics of a cleaned GCE were obtained.

\section{Preparation of RGO/GCE}

The RGO was eletrodeposited on GCE as previously described. ${ }^{11,18}$ Briefly, $0.50 \mathrm{mg} \mathrm{mL}{ }^{-1}$ of graphene oxide suspension (acquired from Sigma-Aldrich, St. Louis, USA) was dispersed in $0.10 \mathrm{~mol} \mathrm{~L}^{-1} \mathrm{Na}_{2} \mathrm{SO}_{4}$, used as supporting electrolyte. By chronoamperometry at applied potential of $-1.5 \mathrm{~V}$ for $1200 \mathrm{~s}, \mathrm{GO}$ was reduced to RGO. The electrode was subsequently dried at room temperature.

\section{Preparation of AuNPs-RGO/GCE}

After the drying of the RGO/GCE, the AuNPs were electrodeposited by cyclic voltammetry at the potential range of 1.3 to $-0.05 \mathrm{~V}$ using a scanning rate of $100 \mathrm{mV} \mathrm{s}^{-1}$ for 25 cycles. After that, the electrode was removed from the electrodeposition solution and washed with deionized water.

Preparation of imprinted isoamyl alcohol on AuNPs-RGO/ GCE

Electropolymerization of MIP was performed by chronoamperometry on the AuNPs-RGO/GCE at potential of $0.75 \mathrm{~V}$ for $120 \mathrm{~s}$ in a solution of $0.10 \mathrm{~mol} \mathrm{~L}^{-1}$ acetate buffer (under $\mathrm{pH}=3.6$ ) as described by Pei et al..$^{19}$ containing $5.0 \times 10^{-4} \mathrm{~mol} \mathrm{~L}^{-1}$ of isoamyl alcohol and $0.050 \mathrm{~mol} \mathrm{~L}^{-1}$ of pyrrole as functional monomer.

After the construction of the MIP-AuNPs-RGO/GCE, the isoamyl alcohol was removed from the polymer matrix to enable the formation of imprinted cavities. To this end, the electrode was immersed in a mixture of acetonitrileacetic acid in a ratio of $5: 1(\mathrm{v} / \mathrm{v})$ for $2 \mathrm{~min}$ at room temperature, followed by washing with deionized water.

For comparison purposes, an electrode denominated NIP (non-imprinted polymer) was developed based on the same conditions in which the MIP-AuNPs-RGO was prepared, but without the addition of the isoamyl alcohol molecule. This was done in order to check the reliability of the measurements.

\section{Experimental measurements}

The rebinding of isoamyl alcohol was carried out through the immersion of the MIP-AuNPs-RGO/GCE in a solution containing $5.0 \times 10^{-6} \mathrm{~mol} \mathrm{~L}^{-1}$ of isoamyl alcohol in $0.10 \mathrm{~mol} \mathrm{~L}^{-1}$ acetate buffer of $\mathrm{pH}=3.6$, under mildly magnetic stirring at $500 \mathrm{rpm}$ for $90 \mathrm{~s}$. After that, the electrode was carefully washed with distilled water to remove the adsorbed physical substances.

Subsequently, the electrode was placed in a threeelectrode conventional cell with MIP-AuNPs-RGO/GCE as working electrode. A solution of $1.0 \times 10^{-3} \mathrm{~mol} \mathrm{~L}^{-1} \mathrm{~K}_{4}\left[\mathrm{Fe}(\mathrm{CN})_{6}\right]$ in $0.10 \mathrm{~mol} \mathrm{~L}^{-1} \mathrm{KCl}$ was chosen as electrochemical active probe to enable us to study the performance of the prepared sensor. Cyclic voltammetry (CV) was recorded between 
-0.20 and $+0.60 \mathrm{~V}$ at a scan rate of $10 \mathrm{mV} \mathrm{s}^{-1}$. Differential pulse voltammograms (DPV) were recorded by scanning at the potential range of 0.0 to $+0.60 \mathrm{~V}$ using modulation amplitude of $50 \mathrm{mV}$, modulation time of $50 \mathrm{~ms}$ and step potential of $4.0 \mathrm{mV}$.

The use of a redox probe was necessary due to the fact that the polymer matrix functions properly in acid solutions while isoamyl alcohol exhibits low electroactivity under acid solutions. Once the imprinted wells are formed, they will provide a path for the diffusion of the redox probe on the surface of the sensor. When the sensor enters into contact with the isoamyl alcohol molecules, the molecules rebound into their complementary cavities, blocking the transfer of electrons from the redox probe to the surface of the electrode. Thus, one will observe a decrease in the intensity of the electrochemical signal, which is inversely related to the concentrations of isoamyl alcohol present in the samples. Bearing that in mind, the analytical data were obtained based on the variation of the current $(\Delta \mathrm{I})$, which was calculated taking into account the oxidation peak currents obtained before and after the combination of the cavities formed with the isoamyl alcohol molecules.

The characterization of the electrochemical sensor was performed by electrochemical impedance spectroscopy (EIS) at potential of $0.22 \mathrm{~V}$ in the frequency range of $0.10 \mathrm{~Hz}$ to $100 \mathrm{kHz}$ and DPV. These techniques are generally used as analytical tools and very useful to characterize the materials in relation to the presence and formation of selective sites, as well as to the adsorptive capacity. ${ }^{20}$ The surface morphology of the electrode was analyzed by field-emission scanning electron microscopy (FEG-SEM; Jeol, model JSM 7500F) operated at $2 \mathrm{kV}$. The microscope is equipped with detector for energy-dispersive $\mathrm{X}$-ray spectroscopy (EDS) analysis.

The limit of detection (LOD), limit of quantification (LOQ) and amperometric sensitivity (As) were calculated according to the equation $\mathrm{LOD}=3.3 \mathrm{SD} / \mathrm{S}$ and $\mathrm{LOQ}=10 \mathrm{SD} / \mathrm{S}$, where SD is the standard deviation of the intercept, and $\mathrm{S}$ is the slope of the calibration curve. ${ }^{21}$

\section{Detection of isoamyl alcohol in fusel oil sample}

Fusel oil was obtained from a sugar and alcohol plant in the region of Araraquara-SP, Brazil. After collection, the fusel oil was stored in an amber bottle and placed in refrigeration. The sample was 100000 -fold dilution with acetate buffer solution, $\mathrm{pH}=3.6$ and the determination of isoamyl alcohol carried out through the standard addition method. The same procedure was adopted for the recovery experiment, where known amounts of standard isoamyl alcohol were added to the samples.

\section{Results and Discussion}

\section{Preparation of MIP-AuNPs-RGO/GCE}

To ensure good adhesion and a greater number of active sites for the MIP-based sensors, RGO and AuNPs were electrodeposited on the surface of the GCE. These nanomaterials are endowed with high electrical properties, surface elevation area, highly sensitive and high surface reaction activity. All these characteristics are important for molecular imprinting. The CV and EIS techniques are an effective tool for monitoring electron transfer on modified electrodes and were used to monitor each step involving the electrode modification. As the isoamylic alcohol molecules do not present electrochemical activity on GCE and GCE modified with AuNPs-RGO in solution of neutral $\mathrm{pH}$, as shown in Figure S1 (Supplementary Information section), the redox probe was employed in this regard. The concentration of $1.0 \times 10^{-3} \mathrm{~mol} \mathrm{~L}^{-1} \mathrm{~K}_{4}\left[\mathrm{Fe}(\mathrm{CN})_{6}\right]$ in $0.10 \mathrm{~mol} \mathrm{~L}^{-1} \mathrm{KCl}$ was used as electrochemical probe to monitor the electrochemical behavior of the sensor during its construction.

In this sense, some typical redox peaks were observed on the bare GCE. When the GCE is modified with RGO an increase was apparently observed in the peak currents, indicating that the electrode has been successfully modified. The increase in current may be attributed to good conductivity and high surface area of graphene sheets. When the RGO/GCE was modified with AuNPs an increase of almost 2 times was noted in the current response compared to the GCE. This increase is related to the size of the nanoparticles, which, in addition to increasing the surface area, facilitate the transfer of electrons through the electrochemical probe, ${ }^{22}$ making the electrode more sensitive.

The electropolymerization process of the pyrrole on the AuNPs-RGO/GCE was performed by chronoamperometry at the potential of $+0.75 \mathrm{~V}$ for $120 \mathrm{~s}$, together with the template molecule in acetate buffer solution $(\mathrm{pH}=3.6)$. DPV was used in this step to investigate the influence of the process. During the electropolymerization process, the isoamyl alcohol molecules are trapped in the polymer matrix because of the ability of the target molecules to interact with the pyrrole units. Theoretically, this interaction occurs between the hydrogen of the hydroxyl group of the molecule template and the nitrogen atom of the $\mathrm{N}-\mathrm{H}$ group of the pyrrole units. ${ }^{18}$

Analyzing the DPV in Figure 1, one will see that, after electropolymerization, the oxidation peak of the redox probe cannot be observed (curve a). This impossibility of visualizing the oxidation peak of the redox probe can be 
associated with the formation of a thin, compact film on the surface of the sensor. With the removal of the isoamyl alcohol molecules from the polymer matrix using a mixture of organic solvents (acetonitrile/acetic acid), the hydrogen bonds between the molecule and the pyrrole units were broken down, leading to the formation of the wells. These cavities should have the same size and positioning as the functional groups of the molecule of interest. Through these channels, the redox probe ions are able to reach the surface of the AuNPs-RGO/GCE, enabling the oxidation of the redox peak. When the MIP-AuNPs-RGO/GCE was placed in a solution containing $5.0 \times 10^{-5} \mathrm{~mol} \mathrm{~L}^{-1}$ of isoamyl alcohol in $0.10 \mathrm{~mol} \mathrm{~L}^{-1}$ phosphate buffer solution (PBS, $\mathrm{pH}=3.6$ ) for $120 \mathrm{~s}$, the peak current was found to decrease (curve c). This shows that some cavities were combined again with isoamyl alcohol molecules, preventing the redox probe from reaching the electrode surface, thus confirming the existence of imprinted cavities in the MIP.

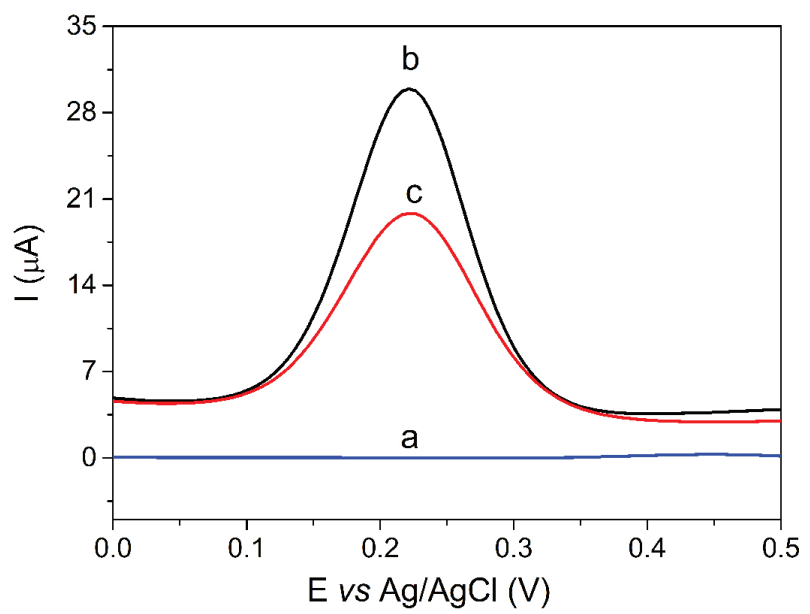

Figure 1. DPV of (a) MIP-AuNPs-RGO/GCE after electropolymerization (b) MIP-AuNPs-RGO/GCE after removal of isoamyl alcohol and (c) MIP-AuNPs-RGO/GCE after $120 \mathrm{~s}$ of rebinding in $5.0 \times 10^{-5} \mathrm{~mol} \mathrm{~L}^{-1}$ isoamyl alcohol solution. All measurements were performed in a solution containing $1.0 \times 10^{-3} \mathrm{~mol} \mathrm{~L}^{-1} \mathrm{~K}_{4}\left[\mathrm{Fe}(\mathrm{CN})_{6}\right]$ in $0.10 \mathrm{~mol} \mathrm{~L}^{-1} \mathrm{KCl}$.

\section{Electrochemical and morphological characterization}

To monitor the electron-transfer resistance on the interface of MIP-AuNPs-RGO/GCE, the EIS was carried out in the frequency range of $0.10 \mathrm{~Hz}$ to $100 \mathrm{kHz}$, as shown in the Nyquist plots (Z" plotted against Z'), using a solution of $1.0 \times 10^{-3} \mathrm{~mol} \mathrm{~L}^{-1} \mathrm{~K}_{4}\left[\mathrm{Fe}(\mathrm{CN})_{6}\right]$ in $0.10 \mathrm{~mol} \mathrm{~L}^{-1}$ $\mathrm{KCl}$ (Figure 2). The typical EIS spectrum is constituted by a semicircular part and a linear part. The semicircular part, which is observed at higher frequency, corresponds to the electron-transfer limited process, while the linear part is associated with the characteristic of the lower frequency range and represents the diffusional limited electrontransfer process. The semicircle diameter is equivalent to the electron-transfer resistance, Rct, which corresponds to the charge transfer kinetics of the redox probe on the electrode surface. ${ }^{23}$

In Figure 2, curve a, one can see the EIS for the bare GCE $(11 \mathrm{k} \Omega)$ and when the MIP sensor is formed on the surface of this bare GCE and before removal of the template molecule, an increase in resistance (curve b) is observed $(21 \mathrm{k} \Omega)$. The increase observed here indicates that the modification may affect the transfer of electrons. Due to the resistance of the compact pyrrole film at the electrode/ solution interface, a barrier is created, and this prevents the redox probe from reaching the electrode surface. However, as can be seen in curve c, the Rct presents a remarkable decrease after the removal of the isoamyl alcohol; this implies that the cavities were formed on the surface of the electrode, where the penetration of the probe $\mathrm{K}_{4}\left[\mathrm{Fe}(\mathrm{CN})_{6}\right]$ was highly likely. In curve d, after rebinding process, an increase is observed in the value of the Rct, which can be attributed to the rebinding of the $5.0 \times 10^{-5} \mathrm{~mol} \mathrm{~L}^{-1}$ isoamyl alcohol molecules in the imprinted cavities, blocking the redox probe from reaching the surface of the electrode.

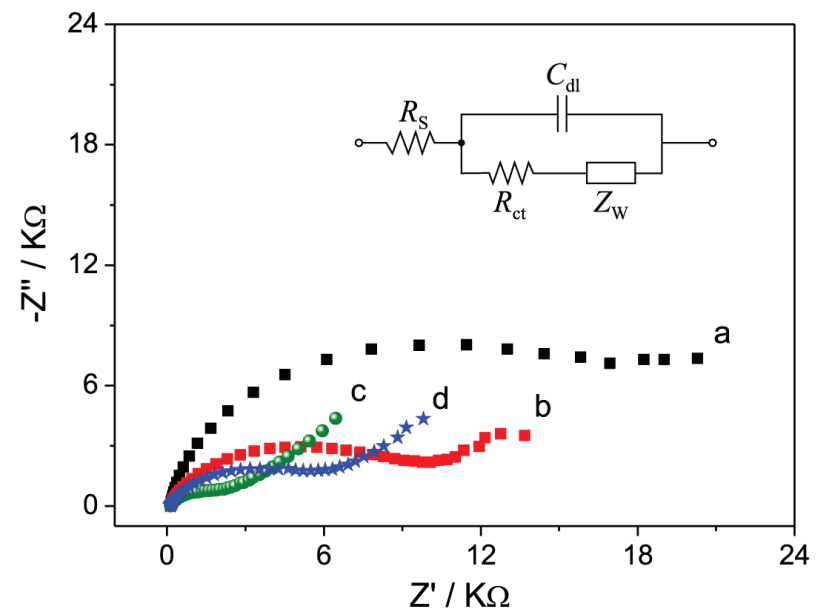

Figure 2. Nyquist diagrams of EIS of (a) bare GCE, (b) MIP-AuNPsRGO/GCE before removal of the template molecule, (c) MIP-AuNPsRGO/GCE after the removal of isoamyl alcohol, (d) MIP-AuNPs-RGO/ GCE after rebinding process in a solution of $1.0 \times 10^{-3} \mathrm{~mol} \mathrm{~L}^{-1} \mathrm{~K}_{4}\left[\mathrm{Fe}(\mathrm{CN})_{6}\right]$ in $0.10 \mathrm{~mol} \mathrm{~L}^{-1} \mathrm{KCl}$, containing $5.0 \times 10^{-5} \mathrm{~mol} \mathrm{~L}^{-1}$ of isoamyl alcohol. Frequency range of $0.1 \mathrm{~Hz}$ to $100 \mathrm{kHz}$.

The morphological characterization of the MIP sensor was performed by field-emission gun scanning electron microscope (FEG-SEM). Figure 3 shows the SEM images of GCE, RGO-GCE, AuNPs-RGO/GCE and MIP-AuNPs-RGO/GCE. In Figure 3b, one can observe the modification of the GCE electrode with the reduced graphene oxide, where the sheets were homogeneously distributed on the surface of the GCE. Figure 3c shows the uniform electrodeposition of the gold nanoparticles, which presented an average diameter of $70 \pm 12 \mathrm{~nm}$, 


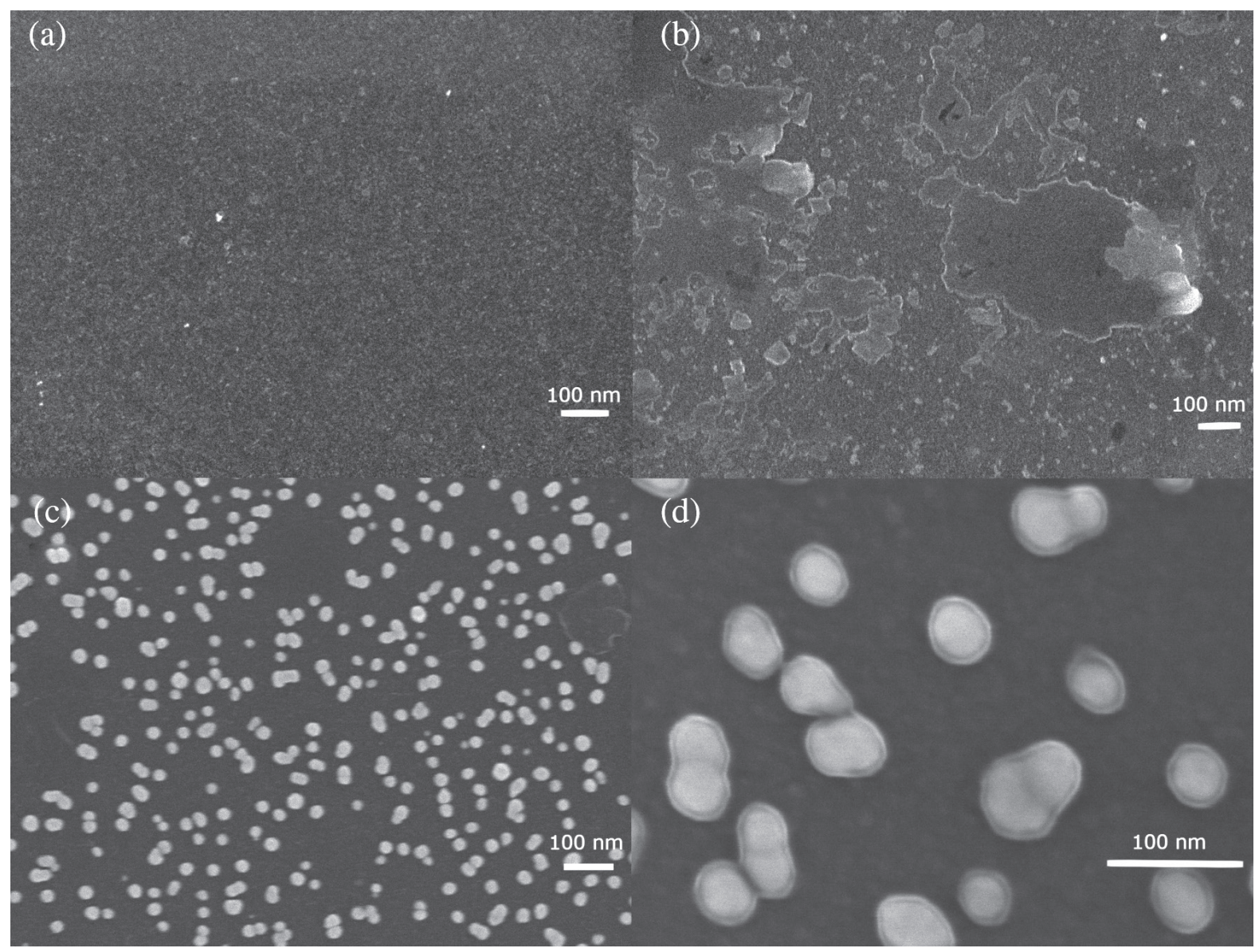

Figure 3. SEM images for (a) GCE, (b) RGO/GCE, (c) AuNPs-RGO/GCE and (d) MIP-AuNPs-RGO/GCE.

distributed over the RGO sheets. Figure S2 (Supplementary Information section) represents the respective EDS, confirming the presence of gold nanoparticles. After electropolymerization to form the MIP on the AuNPs-RGO/ GCE and upon a larger magnification of the image in Figure 3d, one will notice that the MIP encapsulated the AuNPs.

\section{Optimization of conditions that affect electropolymerization}

In general, the thickness of the MIP film directly affects its analytical performance. When the thickness of the MIP film is too thin, the imprinted locations become limited (fewer recognition sites), while too thick film will trap isoamyl alcohol molecules deeply in the polymer matrix, formation of a barrier membrane to the transport of electrons. ${ }^{24}$ Thus, the thickness of the polypyrrole film can be directly controlled by controlling the electropolymerization time and the applicable current or use values. ${ }^{25}$ Furthermore, the concentration of isoamyl alcohol for a certain amount of pyrrole must be analyzed. The amount of isoamyl alcohol is related to the amount of recognition sites that will be formed on the surface of the sensor and can be adjusted by controlling its concentration. Figure $4 \mathrm{a}$ shows the behavior of the current variation $(\Delta \mathrm{I})$ when the isoamyl alcohol concentration varies within the range of $1.0 \times 10^{-5}$ to $0.10 \mathrm{~mol} \mathrm{~L}^{-1}$, keeping the pyrrole concentration always constant. By analyzing the graph, one will note that for the concentration of $0.10 \mathrm{~mol} \mathrm{~L}^{-1}$ pyrrole, the best concentration of isoamyl alcohol required for a good sensor sensitivity is $1.0 \times 10^{-4} \mathrm{~mol} \mathrm{~L}^{-1}$.

For the removal of the isoamyl alcohol molecules attached to the polymer matrix, the use of solvents is one of the most common methods employed. The solvent is capable of swelling the polymer, weakening the hydrogen bonds with the template molecule, causing their release and formation of the cavities. ${ }^{26}$ Removal of the template molecule must be complete in order to ensure good reproducibility and sensitivity of the sensor. In this study, methanol-water, ethanol-water, acetonitrilewater, methanol-acetic acid and acetonitrile-acetic acid were used to remove the template. The results show that acetonitrile-acetic acid when used in the ratio 5:2 (v/v) was able to effectively remove the template molecule while the others were only able to partially dissolve it. Another very important parameter that helps to ensure 
the proper formation of the complementary cavities is the time needed for the MIP sensor to stay in contact with the extraction solvent. Figure $4 \mathrm{~b}$ shows the current variation $(\Delta \mathrm{I})$ in different exposure times of the MIP to the acetonitrile-acetic acid solution 5:2 (v/v). As can be seen, the current rapidly increases with an increase in immersion time up to $60 \mathrm{~s}$. After $60 \mathrm{~s}$, the current is found to decrease, indicating that the excess solvent caused excessive swelling in the polymer, blocking the electroactive surface. Hence, $60 \mathrm{~s}$ was considered the ideal time for the removal of the template molecule.

After the formation of the complementary alcohols, the isoamyl alcohol molecule is formed on the surface of the sensor; hence, the MIP sensor is ready to be used. The time required for the sensor to recognize the molecule of interest was evaluated in $0.20 \mathrm{~mol} \mathrm{~L}^{-1}$ acetate buffer containing $5.0 \times 10^{-6} \mathrm{~mol} \mathrm{~L}^{-1}$ of isoamyl alcohol. The analysis covered the time range of 30 to $180 \mathrm{~s}$. The differential pulse voltammetric responses for the $1.0 \times 10^{-3} \mathrm{~mol} \mathrm{~L}^{-1} \mathrm{~K}_{4}\left[\mathrm{Fe}(\mathrm{CN})_{6}\right]$ in $0.10 \mathrm{~mol} \mathrm{~L}^{-1} \mathrm{KCl}$, were recorded. Figure $4 \mathrm{c}$ shows the effect of the incubation time on the sensor response. The current variation increased up to $120 \mathrm{~s}$, remaining practically stable after this time.
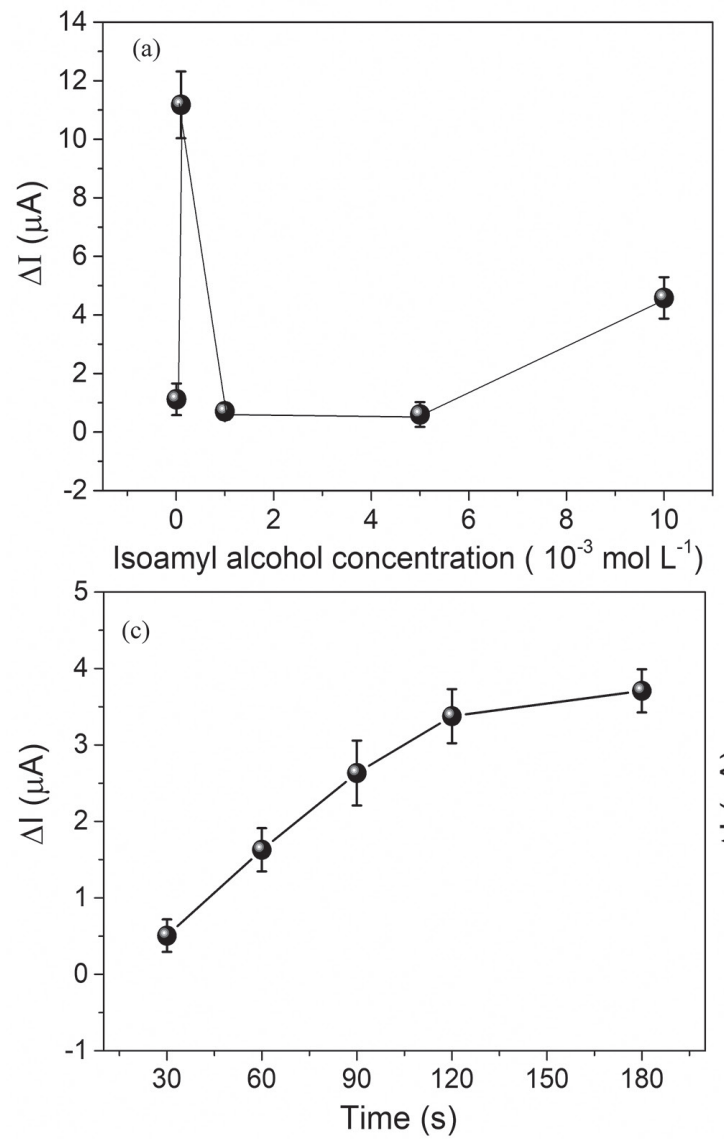

This suggests that the adsorption equilibrium was reached. Thus, $120 \mathrm{~s}$ was chosen as the optimal time for a good sensor response.

In addition, different $\mathrm{pH}$ levels of $0.20 \mathrm{~mol} \mathrm{~L}^{-1}$ acetate buffer solution were tested as a solution for the rebinding of $5.0 \times 10^{-5} \mathrm{~mol} \mathrm{~L}^{-1}$ isoamyl alcohol in the cavities formed. The results shown in Figure $4 \mathrm{~d}$ indicate that the $\mathrm{pH}=3.6$ is the most appropriate for the rebinding process. This $\mathrm{pH}$ is considered reasonable once isoamyl alcohol is soluble in low $\mathrm{pH}$. In addition, in more acidic solutions, pyrrole is found to maintain its properties, not causing damage to the polymeric film.

\section{Electrochemical behavior of the electrochemical active} probe

The kinetic and mechanistic studies of redox probe on the electrode surface were conducted by cyclic voltammetry based on the relationship between the peak current and scan rate $^{27}$ in a solution containing $1.0 \times 10^{-3} \mathrm{~mol} \mathrm{~L}^{-1} \mathrm{~K}_{4}\left[\mathrm{Fe}(\mathrm{CN})_{6}\right]$ and $0.10 \mathrm{~mol} \mathrm{~L}^{-1} \mathrm{KCl}$ on the MIP-AuNPs-RGO/GCE. The scan rate was in the range of 10 to $400 \mathrm{mV} \mathrm{s}^{-1}$. The linear dependence of anodic peak current (Ipa) and cathodic
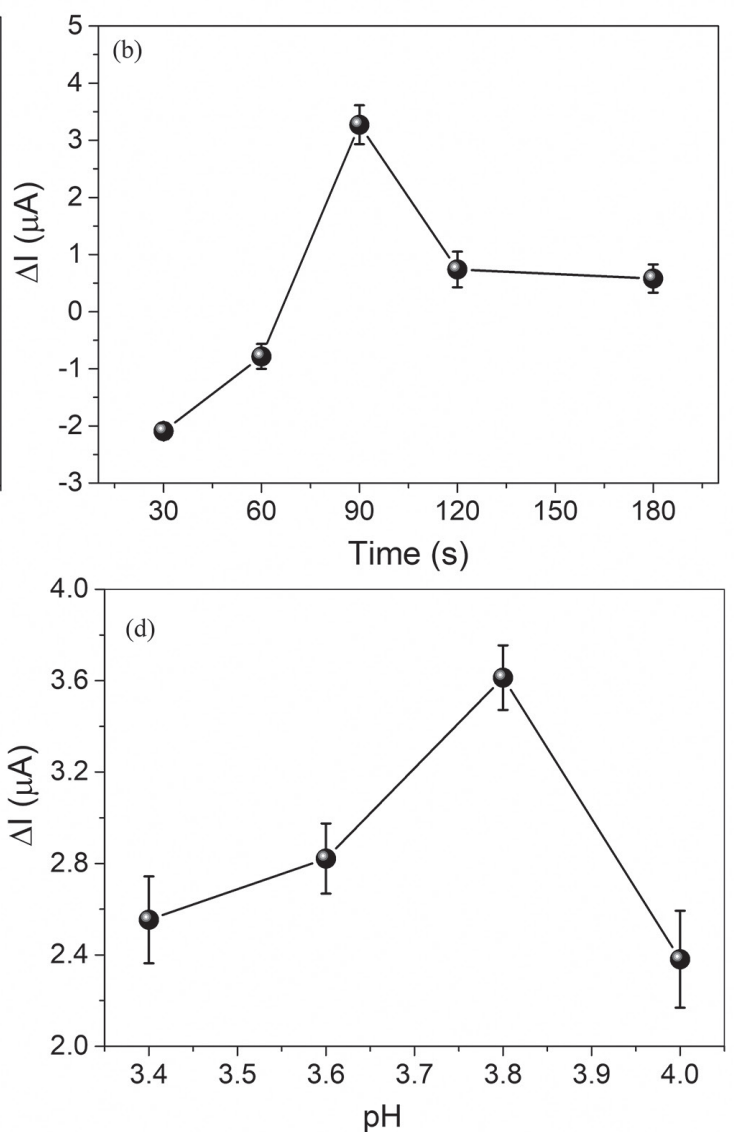

Figure 4. The effect of (a) concentration of isoamyl alcohol, (b) extraction time of molecule, (c) incubation time and (d) pH. The response was measured through DPV in $1.0 \times 10^{-3} \mathrm{~mol} \mathrm{~L}^{-1} \mathrm{~K}_{4}\left[\mathrm{Fe}(\mathrm{CN})_{6}\right]$ with $0.10 \mathrm{~mol} \mathrm{~L}^{-1} \mathrm{KCl}$ supporting electrolyte after rebinding of $1.0 \times 10^{-6} \mathrm{~mol} \mathrm{~L}^{-1}$ isoamyl alcohol. 


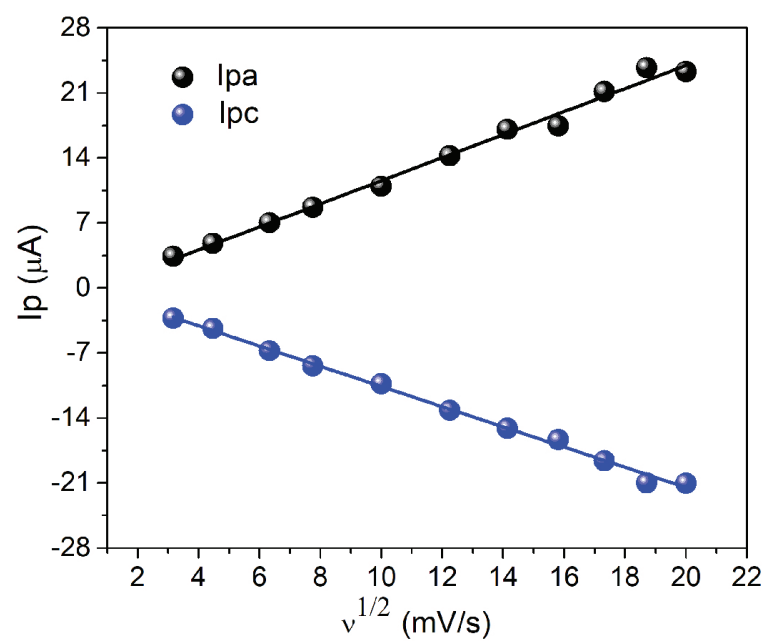

Figure 5. Linear dependence of Ipa and Ipc vs. square root of the scan rate.

peak current (Ipc) (Figure 5) with the root of the speed is expressed as: Ipa $(\mu \mathrm{A})=1.24 \mathrm{v}^{1 / 2}-9.09$ (with correlation coefficient, $R$, of 0.998$)$ and $\operatorname{Ipc}(\mu \mathrm{A})=-1.92 v^{1 / 2}-1.03$ $(\mathrm{R}=0.997)$, showing a mass diffusion-controlled process, which is the ideal case for quantitative determination. ${ }^{23}$

Using the Randles-Sevcik equation (equation 1) and the data obtained from $\mathrm{CV}$ in $\mathrm{K}_{4}\left[\mathrm{Fe}(\mathrm{CN})_{6}\right]$, one can calculate the active area of the GCE, AuNPs-RGO and MIP-AuNPsRGO/GCE.

$\mathrm{Ip}=\left(2.69 \times 10^{5}\right) \mathrm{n}^{3 / 2} \mathrm{ADo}^{1 / 2} \mathrm{v}^{1 / 2} \mathrm{Co}^{*}$

Considering that the current peak by scan rate $\left(\mathrm{Ip} / \mathrm{v}^{1 / 2}\right)$ corresponds to the angular coefficient of the straight lines of the scan rate study, the value for each electrode were: $2.4 \times 10^{-6}$ for GCE, $4.5 \times 10^{-6}$ for AuNPs-RGO and $1.2 \times 10^{-6}$ for MIP-AuNPs-RGO/GCE. The other parameters for $\mathrm{K}_{4}\left[\mathrm{Fe}(\mathrm{CN})_{6}\right]$ in the equation 1 are: $\mathrm{n}$ (number of electrons exchanged during the electron transfer process) equal to 1 , Do (diffusion coefficient) equal to $7.6 \times 10^{-6} \mathrm{~cm}^{2} \mathrm{~s}^{-1}$ and $\mathrm{Co}^{*}$ (concentration of the species in solution) equal to $5.0 \times 10^{-6} \mathrm{~mol} \mathrm{~cm}^{-3}$. So, using the values of the angular coefficients of each electrode in the equation, we can estimate that the A (electroactive area) of each electrode is $0.072,0.134$ and $0.036 \mathrm{~cm}^{2}$ for GCE, AuNPsRGO/GCE and MIP-AuNPs-RGO/GCE, respectively.

These results indicate that when GCE was modified with AuNPs and RGO, the electroactive area increased by almost 1.8 -fold. However, with the formation of the polymeric film on the surface, this value decreases 2-fold in relation to the GCE. This demonstrates that the formation of MIP blocks a significant part of the electroactive area of the sensor, inhibiting the transfer of electrons and/or diffusion of the electroactive species on the surface of the AuNPs/ RGO-GCE electrode. Essentially, it shows the importance of modifying GCE with AuNPs and RGO for the formation of MIP-based sensors.

\section{Thermodynamic analysis}

Adsorption isotherms are able to describe the adsorption capacity and equilibrium relationships between adsorbent and adsorbate, establishing a relationship between the amount of adsorbates adsorbed and the amount of adsorbates adsorbed in solution, considering the equilibrium system and the fixed temperature..$^{28}$

Analyzing the variation of the peak current by the isoamyl alcohol concentration in the range of $1.0 \times 10^{-7}$ to $1.0 \times 10^{-4} \mathrm{~mol} \mathrm{~L}^{-1}$, one will observe that the $\Delta \mathrm{I}$ increases with the amount of analyte up to a maximum limit. This behavior was analyzed in terms of the Langmuir model, which predicts the fractional coverage $\theta$ to vary with adsorbate concentration, $\mathrm{C}$. The relationship is described by equation 2 , where $\theta$ corresponds to ratio $\mathrm{I} / \mathrm{I}_{\max }$, and $\mathrm{I}$ is the current corresponding to concentration $\mathrm{C}$. The peak current had its maximum limit, $\mathrm{I}_{\max }$, in $11.1 \mu \mathrm{A}$.

$\theta=\frac{\mathrm{I}}{\mathrm{I}_{\max }}=\frac{\mathrm{KC}}{1+\mathrm{KC}}$

The data were analyzed by adjusting the experimental data, where $\mathrm{C}$ represents the bulk concentration of the isoamyl alcohol and $\mathrm{K}$ is an equilibrium constant for the adsorption process of molecule in the imprinted sensor. One needs to bear in mind that only the molecules of isoamyl alcohol are adsorbed in the cavities formed on the surface of the sensor. ${ }^{29} \mathrm{~A}$ plot of $\theta$ vs. $\mathrm{C}$ is shown in Figure 6. The value of $\mathrm{K}$ was obtained through the slope by plotting $\mathrm{I}^{-1} v s .[\mathrm{C}]^{-1}$ (shown as an inset in Figure 6), this gives a straight line with a slope of 1.53 , intercept of 0.13 and $\mathrm{R}^{2}=0.997$, which indicates a good agreement with the Langmuir model. Analysis of the slope gives $1.53 \times 10^{6} \mathrm{~mol} \mathrm{~L}^{-1}$ as the value of $\mathrm{K}$, this is within the order of the values found in the literature..$^{28-30}$

\section{Analytical performance of the imprinted sensor}

DPV (Figure 7a) was applied for the quantitative analysis of isoamyl alcohol using the sensor developed under optimized conditions. It is worth noting that DPV is a relatively more sensitive and selective technique compared to the conventional CV. To conduct the analysis, the sensor was placed in acetate buffer solution containing known amounts of the isoamyl alcohol. For each concentration, the period of $120 \mathrm{~s}$ required for rebinding of the molecules in the specific cavities was accounted 


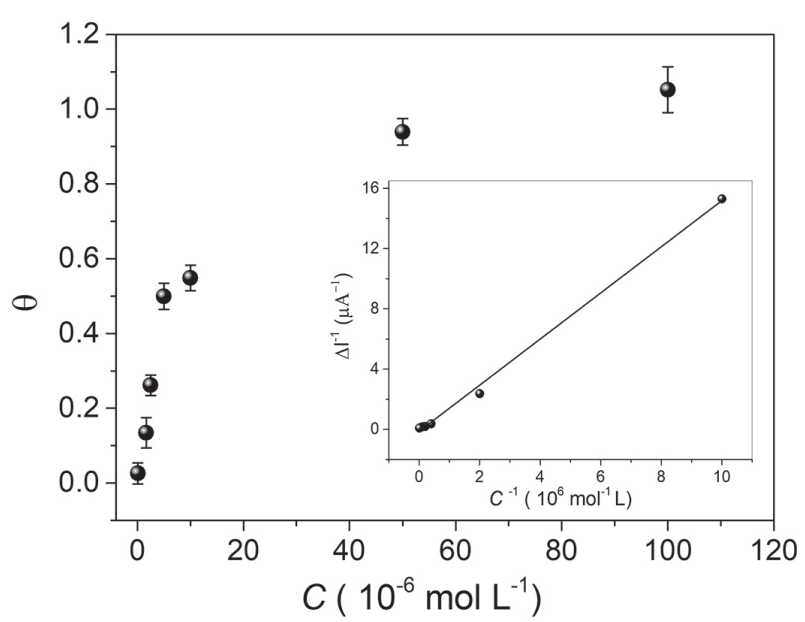

Figure 6. Effect of isoamyl alcohol concentration on the DPV responses in the MIP sensor, where $\theta$ is the fractional surface coverage and $\mathrm{C}$ is the adsorbate concentration. Solution composition, $1.0 \times 10^{-3} \mathrm{~mol} \mathrm{~L}^{-1}$ $\mathrm{K}_{4}\left[\mathrm{Fe}(\mathrm{CN})_{6}\right]$ in $0.10 \mathrm{~mol} \mathrm{~L}^{-1} \mathrm{KCl}$ after rebinding of $1.0 \times 10^{-7}$ to $1.0 \times 10^{-4} \mathrm{~mol} \mathrm{~L}^{-1}$ isoamyl alcohol concentration. The solid line corresponds to the fitting of the data to a Langmuir-type isotherm as obtained by the plot of $1 / \Delta \mathrm{I}$ versus $1 / \mathrm{C}$ (shown as an inset).

for, followed by the detection step. At this stage, as the MIP cavities were partially filled by the isoamyl alcohol molecule, the current, produced by the potassium ferriferrocyanide, was seen to decrease, indicating that fewer cavities were available for the redox probe to reach the electroactive surface. The analytical curve showing the current variation, which relates the difference between the peak current before and after the sensor is immersed in the solution containing isoamyl alcohol, is shown in Figure $7 \mathrm{~b}$. The concentration range studied was $1.0 \times 10^{-4}$ to $1.0 \times 10^{-7} \mathrm{~mol} \mathrm{~L}^{-1}$.

By analyzing the data, it is possible observe that as the amount of isoamyl alcohol increases, the variation of the peak current $(\Delta \mathrm{I})$ also increases until it reaches the plateau at a concentration $(\mathrm{C})$ of $1.0 \times 10^{-5} \mathrm{~mol} \mathrm{~L}^{-1}$. The first range (from $1.0 \times 10^{-7}$ to $5.0 \times 10^{-6} \mathrm{~mol} \mathrm{~L}^{-1}$ ) shown as an inset in Figure $7 \mathrm{~b}$, was used to evaluate the figures of merit with the linear equation: $\operatorname{Ip}(\mu \mathrm{A})=1.1 \mathrm{C}_{\text {isoamyl alcohol }}+2.6 \times 10^{-9}$, with a correlation coefficient of 0.999 , LOD of $8.4 \times 10^{-8} \mathrm{~mol} \mathrm{~L}^{-1}$, LOQ of $2.8 \times 10^{-7} \mathrm{~mol} \mathrm{~L}^{-1}$ and amperometric sensitivity (As) of $1.1 \mathrm{~A} \mathrm{~L} \mathrm{~mol}^{-1}$, (n=3).

To investigate the reproducibility of MIP-AuNPs-RGO/ GCE, three electrodes were prepared under the same conditions for the detection of $1.0 \times 10^{-6} \mathrm{~mol} \mathrm{~L}^{-1}$ of isoamyl alcohol by DPV and each electrode was carried out in three replications. According to the peak current obtained, the relative standard deviation (RSD) calculated was 3.7\%. The electrode was stored in a dry place, protected from light and at room temperature for 10 days. After this period, the electrode was tested again, where $78 \%$ of its initial current was observed (first day), showed that the proposed sensor is stable. An assessment was made regarding the number of extraction and rebinding processes of the template molecule. As the organic solvent swells the polymer matrix, a single use of this process is recommended in order not to damage the specific wells of the molecule, without impairing the selectivity.

\section{Selectivity of the MIP sensor}

The selective recognition of the template molecule is the most important feature of the MIP-based sensors. With the removal of the template from the polymer matrix, cavities are formed with specific binding in a three-dimensional structure, which contributes to the selective identification of the molecule in the MIP sensor.

The selectivity of the MIP-AuNPs-RGO/GCE was evaluated with the addition of the analogs isobutanol and propanol using acetate buffer solution, where the period of $120 \mathrm{~s}$ was taken into consideration for the rebinding of
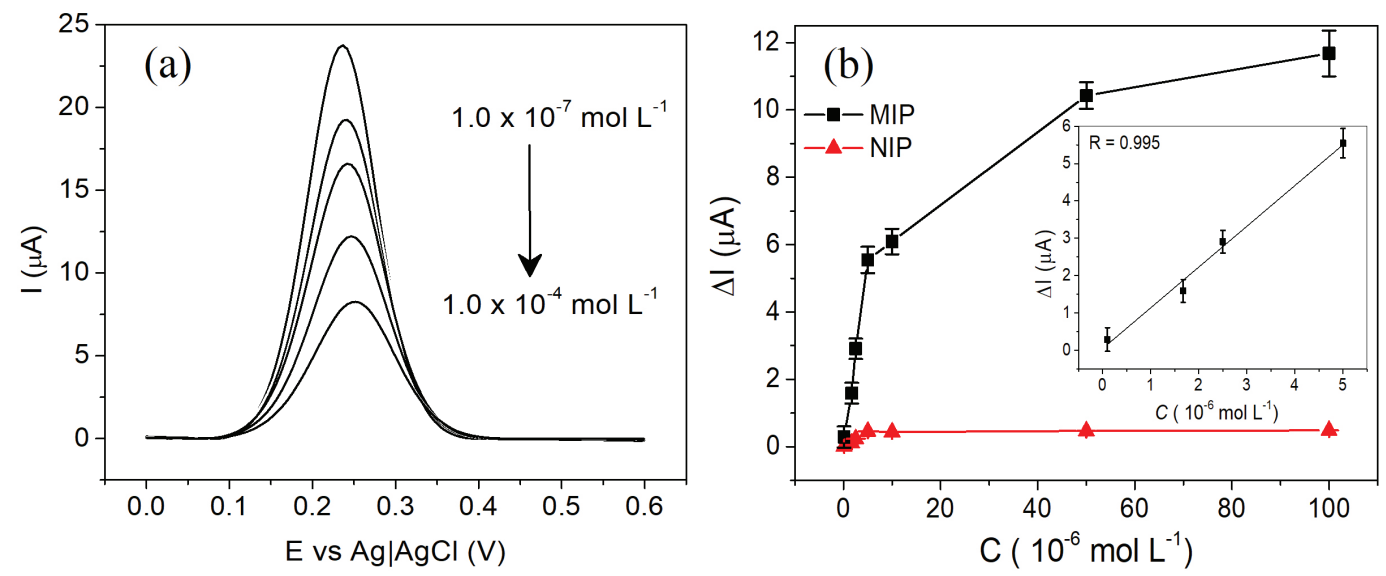

Figure 7. (a) DPV for solution containing $1.0 \times 10^{-3} \mathrm{~mol} \mathrm{~L}^{-1} \mathrm{~K}_{4}\left[\mathrm{Fe}(\mathrm{CN})_{6}\right]$ in $0.10 \mathrm{~mol} \mathrm{~L}^{-1} \mathrm{KCl}$ after the MIP-AuNP-RGO/GCE is placed in solution for rebinding in different concentrations of isoamyl alcohol for $120 \mathrm{~s}$ in $0.20 \mathrm{~mol} \mathrm{~L}^{-1}$ of acetate buffer under $\mathrm{pH}$ of 3.6. (b) Behavior of the relationship between $\Delta \mathrm{I}$ of DPV and isoamyl alcohol concentration and the bottom-left inset demonstrating the calibration curves in the range of $1.0 \times 10^{-7}$ to $5.0 \times 10^{-6} \mathrm{~mol} \mathrm{~L}^{-1}$. 
the molecule. After that, the sensor was washed and placed in $1.0 \times 10^{-3} \mathrm{~mol} \mathrm{~L}^{-1} \mathrm{~K}_{4}\left[\mathrm{Fe}(\mathrm{CN})_{6}\right]$ containing $0.10 \mathrm{~mol} \mathrm{~L}^{-1}$ $\mathrm{KCl}$ and DPV response was obtained. These alcohols were chosen because they are present in considerable concentrations in fusel oil and exhibit structural similarity to the isoamyl alcohol molecule.

As shown in Figure 8, the signal for the MIP sensor (blue columns) was about 6 times larger than that for the NIP sensor (wine columns). For the MIP sensor, an excess of 10-fold of the interferents isobutanol and propanol practically did not change the signal of the peak current in relation to the sensor tested only with isoamyl alcohol (blue columns). This experiment shows that the sensor developed presented good selectivity for isoamyl alcohol even when used in the presence of analogous molecules. For comparison, the wine column refers to NIP that was fabricated following the same procedure, but without isoamyl alcohol. The error bars were obtained from three different experiments conducted in this work.

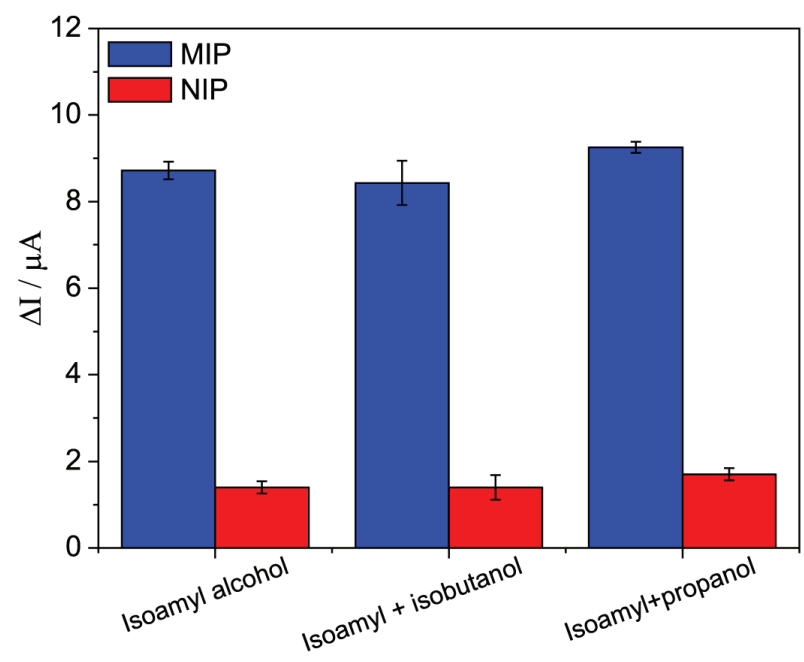

Figure 8. The blue columns are the current change of MIP-AuNPs-RGO/ GCE in acetate buffer solution $(\mathrm{pH}=3.6)$ containing: $1.0 \times 10^{-6} \mathrm{~mol} \mathrm{~L}^{-1}$ of isoamyl alcohol, $1.0 \times 10^{-6} \mathrm{~mol} \mathrm{~L}^{-1}$ of isoamyl alcohol $+1.0 \times 10^{-5} \mathrm{~mol} \mathrm{~L}^{-1}$ of isobutanol and $1.0 \times 10^{-6} \mathrm{~mol} \mathrm{~L}^{-1}$ of isoamyl alcohol $+1.0 \times 10^{-5} \mathrm{~mol} \mathrm{~L}^{-1}$ of propanol. The wine column is the same experiment performed using the non-imprinted sensor (NIP-AuNPs-RGO/GCE).

\section{Practical sample analysis}

In order to examine the applicability of the proposed sensor, the amount of isoamyl alcohol present in fusel oil samples was determined using the spiked recoveries method. In this work, the concentration of isoamyl alcohol was in the range of $2.5 \times 10^{-7}$ to $1.0 \times 10^{-6} \mathrm{~mol} \mathrm{~L}^{-1}$. All measurements were taken in triplicate with different electrodes. The samples were measured by DPV and the results are presented in Table 1 . The measurement results ranged from 97.1 to $109.4 \%$, with SDs ranging from
$1.2-3.9 \%$. The concentration of $255.7 \pm 12.32 \mathrm{~g}$ was found for isoamyl alcohol per liter of fusel oil.

These results indicate that the proposed sensor exhibit good analytical performance, being suitable for the determination of isoamyl alcohol in fusel oil. In addition, it is very important to consider two major factors: the large amount of isoamyl alcohol present in the sample and the large ethanol volume distilled per year. These factors should stimulate the development of technologies capable of exploiting the industrial recovery of isoamyl alcohol.

Table 1. Results of DPV measurements of isoamyl alcohol content in spiked fusel oil samples using the MIP-AuNPs-RGO/GCE

\begin{tabular}{cccc}
\hline Sample & $\begin{array}{c}\text { Added / } \\
\left(10^{-6} \mathrm{~mol} \mathrm{~L}^{-1}\right)\end{array}$ & $\begin{array}{c}\text { Found / } \\
\left(10^{-6} \mathrm{~mol} \mathrm{~L}^{-1}\right)\end{array}$ & $\begin{array}{c}\text { Recovery } \\
(\text { mean } \pm \mathrm{SD})^{\mathrm{a}} / \%\end{array}$ \\
\hline \multirow{3}{*}{ Fusel oil } & 0 & $3.7 \pm 0.3$ & - \\
& 0.25 & $0.25 \pm 0.05$ & $100 \pm 20$ \\
& 0.50 & $0.52 \pm 0.02$ & $104 \pm 2$ \\
\hline
\end{tabular}

${ }^{\mathrm{a}} \mathrm{n}=3$. SD: standard deviation.

\section{Conclusions}

In this paper, an electrode modified with AuNPs and RGO was used for the formation of an electrochemical alcohol isoamylic-imprinted sensor, via electropolymerization of pyrrole. The reduced graphene oxide combined with the gold nanoparticles were fundamental for the good performance of the sensor. The good performance displayed by the sensor is attributed to the increase of the conductive surface area, which contributed towards enhancing the electron transport and providing more room for alcohol isoamylic molecule immobilization per unit surface area. This led to a higher selectivity and sensitivity of the MIP sensor. The electropolymerization process was very simple, rapid, and controllable. Under the optimum conditions, the MIP-AuNPs-RGO/GCE showed low limit of detection and quantification, good repeatability and stability. In addition, the sensor exhibited high selectivity relative to the target molecule even when the solution contained molecules with similar structures. The high percentage of the recovery (between 96 and 110\%) indicates that the proposed method has excellent degree of accuracy, being essentially suitable for the determination of isoamyl alcohol in fusel oil, without the use of derivatization or laborious sample preparation.

\section{Supplementary Information}

Supplementary information is available free of charge at http://jbcs.sbq.org.br as PDF file. 


\section{Acknowledgments}

The authors would like to thank the São Paulo Research Foundation (FAPESP, process number 2017/23529-6 and 2018/12131-6) and Coordination for the Improvement of Higher Education Personnel (CAPES) for the financial support granted in the course of this research.

\section{References}

1. Kamani, M. H.; Eş, I.; Lorenzo, J. M.; Remize, F.; Roselló-Soto, E.; Barba, F. J.; Clark, J.; Mousavi Khaneghah, A.; Green Chem. 2019, 21, 3213.

2. Dörmő, N.; Bélafi-Bakó, K.; Bartha, L.; Ehrenstein, U.; Gubicza, L.; Biochem. Eng. J. 2004, 21, 229.

3. Awad, O. I.; Mamat, R.; Ibrahim, T. K.; Kettner, M.; Kadirgama, K.; Leman, A. M.; Saiful, A. I. M.; Renewable Energy 2018, $118,858$.

4. Pereira, F. S.; Pereira, L. J.; Crédito, D. F. A.; Girão, L. H. V.; Idehara, A. H. S.; González, E. R. P.; RSC Adv. 2015, 5, 81515.

5. Pérez, E. R.; Cardoso, D. R.; Franco, D. W.; Quim. Nova 2001, $24,10$.

6. Neale, M. E.; J. Chromatogr. A 1988, 447, 443.

7. LaCourse, W. R.; Johnson, D. C.; Rey, M. A.; Slingsby, R. W.; Anal. Chem. 1991, 63, 134.

8. Rawool, C. R.; Srivastava, A. K.; Sens. Actuators, B 2019, 288 , 493.

9. Duan, D.; Yang, H.; Ding, Y.; Li, L.; Ma, G.; Electrochim. Acta 2019, 302, 137.

10. Tonelli, D.; Ballarin, B.; Guadagnini, L.; Mignani, A.; Scavetta, E.; Electrochim. Acta 2011, 56, 7149.

11. Beluomini, M. A.; da Silva, J. L.; Sedenho, G. C.; Stradiotto, N.; Talanta 2017, 165, 231.

12. Whitcombe, M. J.; Chianella, I.; Larcombe, L.; Piletsky, S. A.; Noble, J.; Porter, R.; Horgan, A.; Chem. Soc. Rev. 2011, 40, 1547.
13. Ratinac, K. R.; Yang, W.; Gooding, J. J.; Thordarson, P.; Braet, F.; Electroanalysis 2011, 23, 803.

14. El Jaouhari, A.; Yan, L.; Zhu, J.; Zhao, D.; Khan, M. Z. H.; Liu, X.; Anal. Chim. Acta 2020, 1106, 103.

15. Pereira, T. C.; Stradiotto, N. R.; Microchim. Acta 2019, 186, 764.

16. Beluomini, M. A.; Karimian, N.; Stradiotto, N. R.; Ugo, P.; Sens. Actuators, B 2019, 284, 250.

17. Parate, K.; Karunakaran, C.; Claussen, J. C.; Sens. Actuators, B 2019, 287, 165.

18. Beluomini, M. A.; da Silva, J. L.; Stradiotto, N. R.; Microchim. Acta 2018, 185, 170.

19. Pei, Q.; Qian, R.; J. Electroanal. Chem. 1992, 322, 153.

20. Pereira, A. C.; Braga, G. B.; Oliveira, A. E. F.; Silva, R. C.; Borges, K. B.; Chem. Pap. 2019, 73, 141.

21. Benedito da Silva, O.; Machado, S. A. S.; Anal. Methods 2012 , 4, 2348.

22. Sanghavi, B. J.; Wolfbeis, O. S.; Hirsch, T.; Swami, N. S.; Microchim. Acta 2015, 182, 1.

23. Bard, A. J.; Faulkner, L. R.; Electrochemical Methods: Fundamentals and Applications; $2^{\text {nd }}$ ed.; Wiley Global Education: New York, 2001.

24. Duan, D.; Si, X.; Ding, Y.; Li, L.; Ma, G.; Zhang, L.; Jian, B.; Bioelectrochemistry 2019, 129, 211.

25. Schirmeisen, M.; Beck, F.; J. Appl. Electrochem. 1989, 19, 401.

26. Kamalodin Kor, K.; Talanta 2016, 146, 181.

27. Wang, J.; Analytical Electrochemistry, $3^{\text {rd }}$ ed.; John Wiley \& Sons: New Jersey, 2006.

28. Abiman, P.; Wildgoose, G. G.; Crossley, A.; Compton, R. G.; J. Mater. Chem. 2008, 18, 3948.

29. Agra-Gutiérrez, C.; Compton, R. G.; Electroanalysis 1998, 10, 204.

30. Chen, L.; Li, X.; Tanner, E. E. L.; Compton, R. G.; Chem. Sci. 2017, 8, 4771.

Submitted: April 2, 2020 Published online: August 26, 2020 